\title{
Timescapes: Design and Additive Manufacturing Workflows for freeform, ornamental architectural surfaces
}

XXIV International Conference of the Iberoamerican Society of Digital Graphics Medellin | Colombia

\author{
Felix Raspall \\ Adolfo Ibanez University | Chile | felix.raspall@uai.cl \\ Carlos Banon \\ Singapore University of Technology and Design | Singapore | carlos_banon@sutd.edu.sg \\ Sourabh Maheshwary \\ Singapore University of Technology and Design | Singapore | carlos_banon@sutd.edu.sg
}

\begin{abstract}
The application of Additive Manufacturing in architecture is an emerging research trend. Most of the research focuses on large-scale concrete and clay printing and, however, overlooks Fused Filament Fabrication (FFF), the most ubiquitous and inexpensive printing technology. Reasons for this include FFF's reduced build volume, low mechanical resistance, and long printing times. In this research, the use of FFF for the construction of ornamental architectural surfaces is proposed, developed, and tested in a permanent, full-scale project. A discussion on the digital workflows, design, manufacturing, and assembly processes is presented, leading to a conclusion and outlook based on the evidence collected and highlighting the key advantages and main challenges of using FFF in architecture.
\end{abstract}

Keywords: Additive manufacturing; Freeform architecture; Ornament; Fused filament fabrication

Parametric architecture

\section{INTRODUCTION}

The development of freeform modeling and parametric design tools has supported the design of architectures of increasing geometric and ornamental complexity. However, the manufacturing of intricate designs continues to be a significant challenge. The use of CNC subtractive methods has offered the first fabrication approach for freeform and highly ornamental components (Manahl et al., 2012). Still, with subtractive methods, as articulation and curvature increase, it quickly translates into a longer time, higher cost and more waste. In this context, Additive Manufacturing (AM) emerges as a real technological alternative, with which adding complexity does not directly translate into increased time, cost, and material use.

Additive Manufacturing in architecture has been applied primarily for representation purposes in scale models. Progress towards implementing AM to functional architectural components is an emerging research domain, primarily on large-scale robotic printing, and in space frame structures. However, Fused Filament Fabrication (FFF), the most widespread and affordable printing technology, remains understudied as a viable construction method. The few existing examples have been for short-term exhibitions and published in design media but not discussed in academic papers, therefore, with limited academic contributions.

The main objective of this research is to provide evidence of the potentials and limitations of FFF for the creation of freeform, ornamental architectural spaces. Timescapes is a design research project that develops a digital workflow for low-cost FFF technology for long-term, one-to-one architectural piece. The project follows the design, manufacturing, and assembly stages, developing the necessary tools and workflows. A built $140 \mathrm{~m} 2$ pavilion serves as a one-to-one proof of concept of the practical use of additive manufacturing in architecture. This paper provides a detailed description and discussion on the digital design and manufacturing processes for a large-scale project with a 20 -year lifespan.

\section{BACKGROUND}

The application of additive manufacturing in construction is an emergent field, promoted by the lowering costs, and increased speed, size, and strength of printed parts (Craveiroa et al., 2019). Multiple AM technologies are being researched for architectural applications, including Concrete Extrusion Printing, Clay Extrusion Printing, Wire Arc Additive Manufacturing and Direct Metal Laser Sintering.

However, the most widespread and affordable AM technology, Fused Filament Fabrication, has seen limited research efforts. FFF consists of the extrusion of a fused polymer filament through a CNC controlled nozzle, building of the part layer by layer. Key advantages of this technology are its relatively low cost, ease of production and availability. Its drawbacks include low printing speed, relatively low mechanical properties of parts, need for supports on steep cantilevers and bridges, and a comparatively lower resolution. 


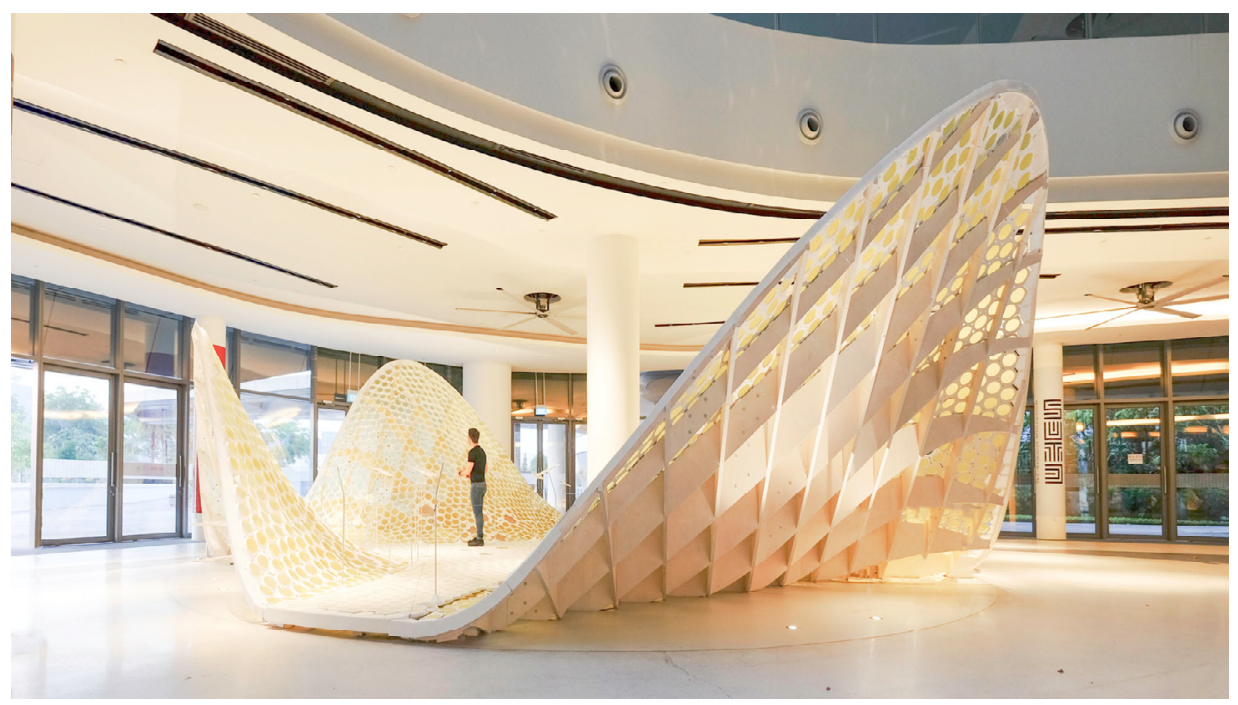

Figure 1: Exterior view of Timescapes.

At first glance, its mechanical and size limitations make FFF incompatible with architectural applications. Therefore, the use of FFF in architectural projects has been limited. Some experimental pavilions are valuable precedents, including the Vulcan Pavilion (Domus, 2015), the EGG Project (Hipolite, 2014), The Rise Pavilion (DeFacto 2016), and (ultra) Light Network (Banon and Raspall, 2020). In addition, most of the research and creative work on FFF in construction has been for temporary structures and has not been published as scientific studies.

\section{METHODOLOGY}

The methodology follows a design research sequence, carefully developing, executing, and documenting all stages of the process. It develops the complete digital and manufacturing workflow from conceptual design to fully built project. The main steps in the research include:

- Creation of initial concept design and the basic form of the project.

Development of a functional parametric model, which integrates geometric constraints from the conceptual design and manufacturing constraints from the AM process. These constraints include maximum build volume, no-support requirements, budget and production time.

- Planning of the manufacturing sequence and supply chain for the unique components.

- Creation of an operative assembly strategy for the assembly of thousands of unique components into a single project, executed by labor with no digital expertise.

Development of recommendations for architectural projects that use additive manufacturing

\section{TIMESCAPES}

Timescapes is the 10th anniversary pavilion for Singapore University of Technology and Design (SUTD). Its form was conceived from a circular plan, where four points are elevated to create a 3D freeform surface. The resulting geometry defines an interior space with four entrances. Inside of the pavilion, an exhibition of a collection of objects is displayed. The double-curved geometry presents a manufacturing challenge as it is not a developable surface and cannot be directly built from flat material. Figure 1 shows the project's overall geometry.

The surface was used as the base to develop a design-tomanufacturing process tailored to the use of FFF technology. The details of the design, manufacturing and assembly processes are described in the following subsections.

\section{DESIGN}

The design process starts with the concept of a curved circular geometry with an edge that lifts in four points. The periodic curve references the idea of time, which connects with the purpose of the structure as a time capsule. Figure 2 shows Timescapes' overall geometry. The diameter of the design is 12 meters.

The resulting double-curved surface was used as a base for the technical development of the project. The surface was subdivided into three main components:

- $\quad$ The loadbearing structure, made of plywood ribs with a waffle structure,

The ornamental surface, made of $4000+$ unique $3 \mathrm{D}$ printed tiles, located on the non-flat parts of the surface

The floor, consisting of perforated plywood panels, and is located on the flat areas of the structure.

A set of computational processes were created to develop the project technically. First, the original surface was divided into a flat section, which was used to design the floor panels, four curved sections, or wings used to design the tiles. Then, the four wings were tessellated using a constraint of $200 \times 200 \times 100 \mathrm{~mm}$ bounding box, which was used to secure that each component is printable in an 


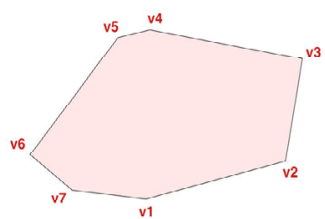

1

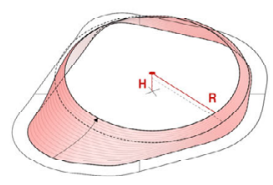

5

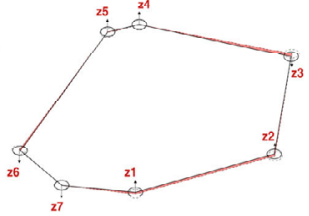

2

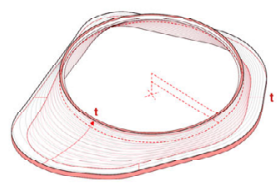

6
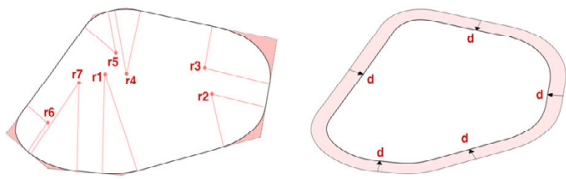

3
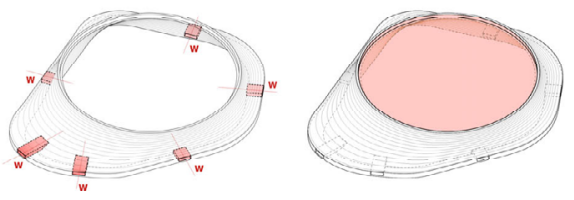

7

Figure 3: Generative process for tile components.

entry-level FFF printer. The tessellation algorithm involved the closest circle packing, which provided an evenly distributed set of points on the surface, from which a Voronoi subdivision was derived.

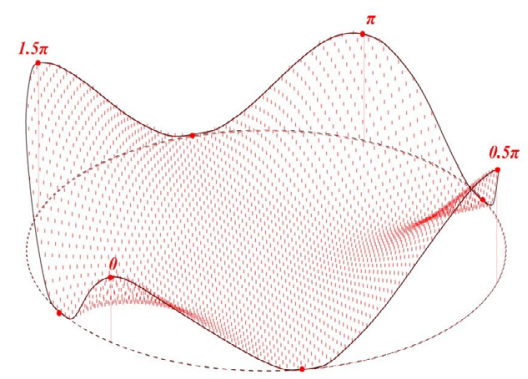

Figure 2: Exterior view of Timescapes

The geometric design of each tile, shown in Figure 3, can be summarized in eight steps. First, the polygonal geometry of the base of the tile comes from the Voronoi subdivision process (1). Then, the polygon is planarized to the best fitting plane (2). The corners are filleted and offset to create the bottom of the tile (3). A circle is created in the centroid of the tile, elevated a height that is proportional to the tile's vertical position in the project (4). The tiles that are located higher have a taller neck (5). Two loft surfaces are created, giving the tile its thickness (6). Then, connection holes are subtracted from the design (7). Once the tiles were printed, circular, translucent plexiglass is placed over the circular rim of the tile (8). The tile design was conceived to be printable without support, reducing the printing time, cost, and waste. In addition, a unique ID number was embossed on the tile.

A particular tile was designed for the edges of the surface. The design has two requirements. First, it provides support for the printed surface, which hangs from the edge. Second, it provides the geometric end of the pattern. The final design used with a variation of the algorithm, which detected those cells in the tessellation that belong to an edge and generated the tile geometry. The edge tiles have two ducts that ran along the edge and were designed to house two continuous aluminum tubes that reinforce the structure of the tiles. Figure 4 shows a detail of the edge tile.

The plywood structure was designed using the $12 \mathrm{~m}$ diameter base surface, from which a $500 \times 500$ grid was superimposed to determine the location of the waffle structure. The ribs were designed computationally, including the finger joint detail and the ID numbers. The depth of ribs was variable, following the structural requirements. On the edges, the thickness was minimal, of $50 \mathrm{~mm}$, while towards the center, the structural depth increased to around $500 \mathrm{~mm}$

The floor panels were designed to replicate the pattern of the printed surface. The closest packing of circles was created, from which a filleted Voronoi pattern was derived. The circular holes were made to receive translucent plexiglass, giving the printed surfaces and the floor visual continuity despite their different materiality. The subdivision of the floor surface into panels was done to fit the 4'x8' size of the stock, using the cells as a division curve between panels to disguise the seams.

\section{MANUFACTURING}

Timescapes was designed, manufactured, and assembled over 5 months. Therefore, careful planning of manufacturing was essential. The three main components of the project (structure, floor, and printed tiles) were commissioned to different specialized vendors.

The printed surface was the most challenging task because of its novelty. With more than 4,000 unique pieces, these needed to be correctly designed and printed in the right sequence, to be able to be produced and installed on time.

The tiles were printed in an FFF printing farm with 50 printers running on parallel, $24 / 7$ for four weeks. The tile design was iterated several times, to ensure that they are all printable within the limitations of the printing bed and without supports. Besides, a printing time of fewer than five hours per tile was crucial to ensure that three shifts could be done in a single day. 


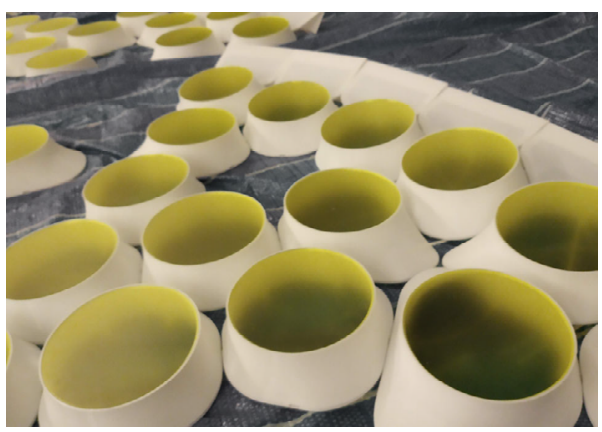

Figure 5: Assembly of the tiles into "large chainmail".

The production was divided into batches, which were delivered on a bi-weekly basis. The sequencing of the printing process was done based on wings. This process ensured that the tiles were delivered in order, and could be assembled as soon as received, limiting the stock of parts in the construction site and facilitating assembly.

The tiles had a filename and printed ID that facilitated the positioning of the tiles in the system. The ID system followed this pattern: cXXrYYmZZ, where the ' $c$ ' and ' $r$ ' indicated the column and row based on the structural waffle grid, while the ' $m$ ' indicating the location of the tile within the quadrant. Figure 5 shows the tiles over a template, showing the assembly in process.

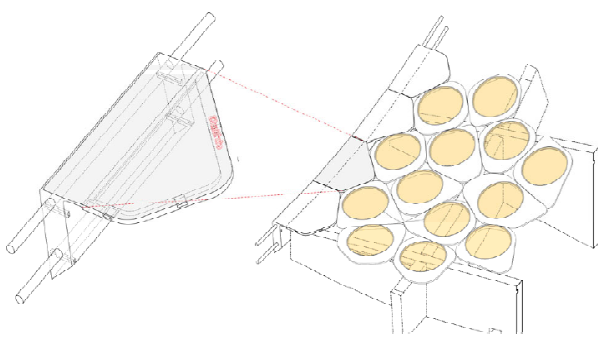

Figure 4: Design of the edge tile.

The material for the prints was white Polylactic acid filament, which offers a reliable printing quality and is widely available. Besides, PLA is a bioplastic produced from renewable resources, which increases the sustainability of the project. Being in an interior space, the exposure to UV, which can degrade this polymer, was minimal. The plywood structure supported the tiles, so the structural requirements of each tile were limited to their own weight hanging from the edge tiles. The printing parameters are specified in Table 1.

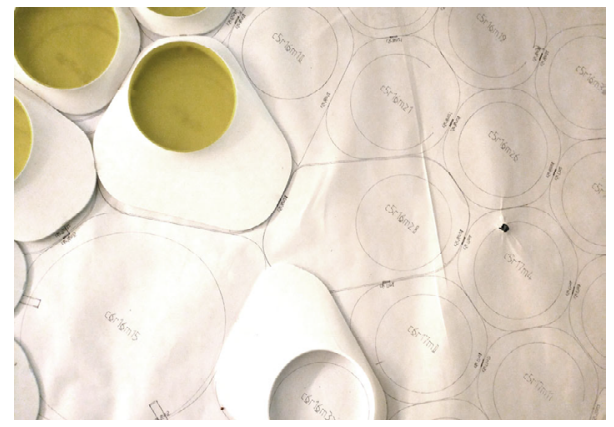

Figure 6: Template used to position all tiles in order.

Table 1: Printing parameters for the tiles.

\begin{tabular}{ll}
\hline Parameter & \\
\hline Nozzle Temperature & $218^{\circ}$ \\
Bed Temperature & $60^{\circ}$ \\
Layer Height & $0.2 \mathrm{~mm}$ \\
Printing Speed Walls & $60 \mathrm{~mm} / \mathrm{s}$ \\
Printing Speed Infill & $70 \mathrm{~mm} / \mathrm{s}$ \\
Infill Density & $10 \%$ \\
Infill Pattern & Gyroid \\
\hline
\end{tabular}

\section{ASSEMBLY}

The assembly process consisted of three key phases: the erecting of the wooden structure, the cladding layer's addition, and the flooring.

Firstly, a set of 24 parallel wooden ribs was temporally fixed over the floor. Once completed, the second set of wooden profiles formed, the $500 \times 500 \mathrm{~mm}$ waffle.

Secondly, the 3D Printed cladding layer was "draped" over the structure's four raised regions (Fig. 7). Due to the high number of components involved in cladding each wing, a unique ID was imprinted in every tile, referencing each part to the whole. Once the tiles were identified, they were placed according to the printed 1:1 template. By using this method, the pre-assembly of the cladding layer was expedited, and positioning errors were prevented. For each of the tiles, a circular piece of transparent yellow acrylic was glued.

Simultaneously to the four wings' pre-assembly on the floor, the installation of the edges was performed. A specially designed set of L-shaped tiles were produced to confer additional rigidity to the system. Two concealed $8 \mathrm{~mm}$ diameter metal rods run through the whole perimeter, increasing the stiffness and impact resistance. The attachment of the edge to the structural ribs was achieved using zip-ties. Once the full edge was adjusted and secured, the installation of the four wings of 3D printed tiles was performed. First, the cladding layer of tiles was presented on top of the wooden waffle and temporally secured to the structure. Then, the upper tiles were connected to the edge. Successive punctual tightening of the connections was applied, guiding the cladding to its final geometry.

Lastly, a set of 25 plywood CNC-ed boards were directly positioned and fixed onto the structure. Due to the high 


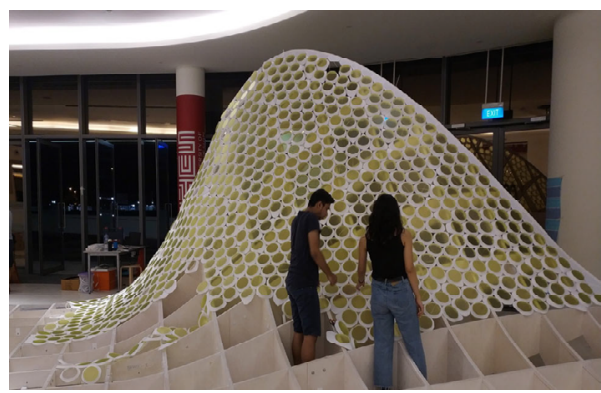

Figure 7:. Installation of the wing tiles.

level of geometric accuracy obtained in the 3D printed fabric, the fitting of components was very precise (Fig. 8).

\section{RESULTS AND DISCUSSION}

The results of the project demonstrate that FFF is a viable technology for complex, freeform and ornamental spaces in architecture.

Timescapes overcame the critical limitations of AM: print speed and print volume. It did so by taking advantage of the widespread availability of FFF printing services due to the equipment's low-cost. In Timescapes, with a price per

tile of around 10 US dollars, the cost per square meter of printer surface is $320 \$ / \mathrm{m} 2$, comparable to other massproduced cladding solutions such as aluminum cladding systems.

\section{KEY ADVANTAGES}

The prototype evidenced the following benefits of FFF in producing ornamental surfaces:

- $\quad$ FFF facilitates the materialization of unique and complex ornamental surfaces with a $320 \$ / \mathrm{m} 2$ cost.

- It enables freeform surfaces, which may be difficult and wasteful to materialize with other manufacturing technologies.

It provides a viable solution to manufacture intricate, computer-generated ornamental patterns with few geometric constraints.

Low-cost and widespread availability of FFF printing services (at least in Singapore, United States and Chile, where the research team has built projects) enable the production of large volumes of components.

\section{MAIN CHALLENGES}

The prototype also identified the following limitations of FFF for ornamental surfaces:

- The low mechanical performance of polymers limits the applicability of FFF to non-structural components such as ornamental surfaces.

FFF viability relies on printing farms with several printers running on parallel. Still, it took 4 weeks to produce around $100 \mathrm{~m} 2$ of printed surface, so, larger projects would require significantly larger production infrastructure than what is available to date.

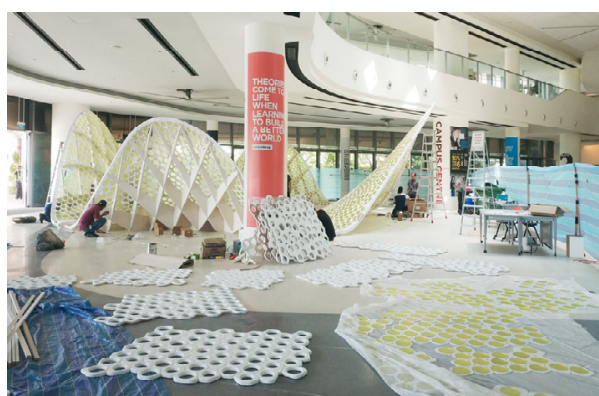

Figure 8: Installation of floor panels

Sustainability concerns with polymers in general exist. Although FFF produces very limited waste, the final fate of plastics is a concern.

\section{DESIGN RECOMMENDATIONS}

The results of this project and its successful implementation into a permanent, built structure can be compiled into a set of recommendations for designers working on similar projects. These are summarized in the following points:

- The parametric model should keep track of the building volume of all printed components, which can be easily implemented with a minimum bounding box calculator. In Timescapes, the few parts that required printing and gluing were the most problematic, so it is essential to identify them early in the design process.

If possible, designs that require no support are preferred. These take time to print, require postprocessing, and create waste. A simple verification can be implemented in the parametric model by determining the angle of each face and comparing it with the maximum cantilever angle. While this is not $100 \%$ certain, it provides a good indication to spot components that are prone to have printing issues.

The geometric freedom of AM allows the design of custom connection details. It is important to develop and prototype these details early on, as they will have a significant impact on the assembly sequence.

Include a well-thought ID system that helps identify and position the components in space. When having an extensive collection of unique elements, finding one part in the whole can be challenging and time-consuming.

Find a production sequence where adjacent parts are printed at the same time so that while the printing is happening, the assembly can take place.

\section{ACKNOWLEDGMENTS}

We would like to thank the greatly supportive team behind this project, including SUTD community and leadership for the support along the way. We are grateful to our AirLab team, including Natalie Chen, Muhd Syahid Mustapa, Sihan Wang, Michele Sodano, Aurelia Chang, Lui Chi, Huang Kunsheng, Luo Quihuan, Megan Chor, Tai YuJie, Kwang KayJie. We are thankful to Chiu Po Heng, Darius Chow De Wei who have produced all printed components with all the flexibility and responsiveness needed. 


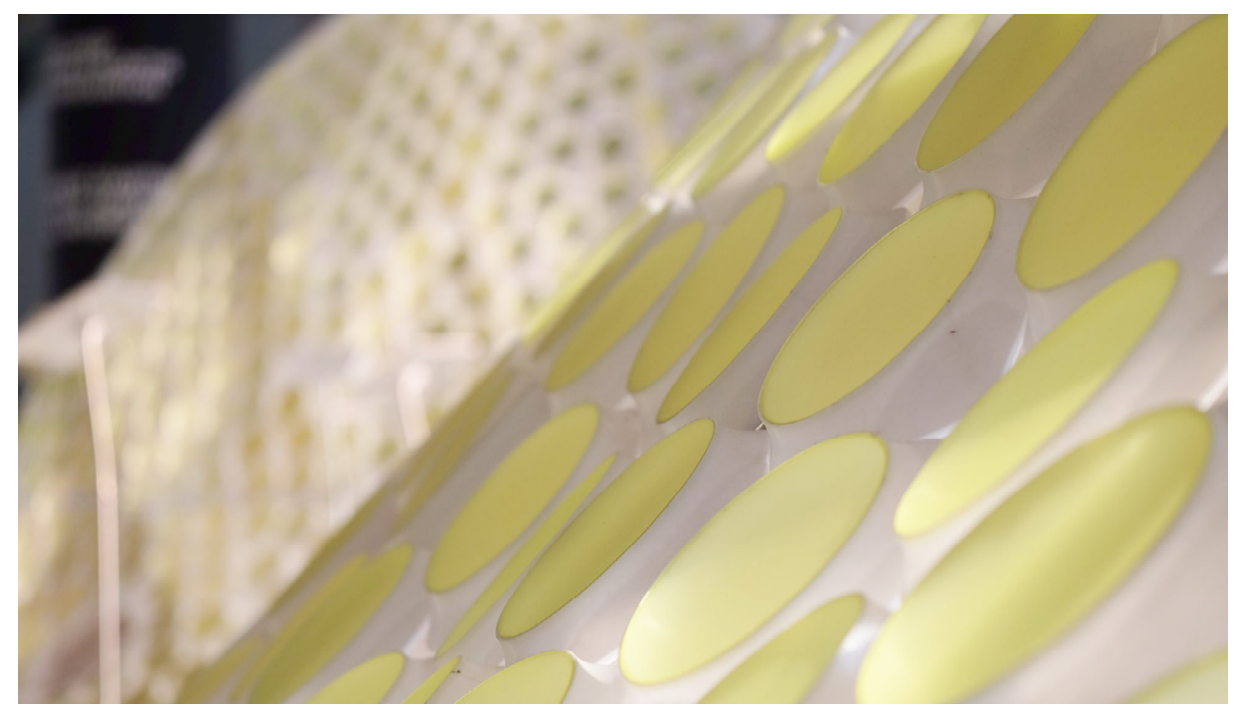

Figure 9: Assembly of the tiles into a "large chainmail".

\section{REFERENCES}

Banon C and Raspall F. "Systems Integration:(ultra) Light Network." 3D Printing Architecture. Springer, Singapore 8998.

Craveiroa, F., Duartec, J. P., Bartoloa, H., \& Bartolod, P. J. (2019). Additive manufacturing as an enabling technology for digital construction: A perspective on Construction 4.0. sustainable development, 4,6 .

DeFacto (2016) Guinness World Record: Largest 3D Printed Structure $2016 \quad$ Retrieved from https://uploads.strikinglycdn.com/files/069b1391-3fc3-477fae99-131fc230137f/DeFacto\%20-\%20Rise\%20Pavilion \%20$\% 20$ Media\%20Kit.pdf
Domus (2015) Vulcan Retrieved from https://www.domusweb.it/en/news/2015/10/10/laboratory_for creative_design_vulcan.html

Hipolite, W. Project Egg (2014) Retrieved from https://3dprint.com/17680/project-egg-3d-printing/

Manahl, M., Stavric, M., \& Wiltsche, A. (2012). Ornamental discretisation of free-form surfaces: Developing digital tools to integrate design rationalisation with the form finding process. International journal of architectural computing, 10(4), 595612.

Raspall, F and Banon C. "3D Printing Architecture: Towards Functional Space Frames." In Proceedings of the 23rd CAADRIA Conference, 2018. 